\title{
Évaluation du risque de liquéfaction de la fondation d'un barrage en terre à partir d'essais in situ
}

\section{M.R. EL OUNI} I. GUETTAYA

Département de Génie rural INAT - Tunis

43, avenue Charles-Nicolle 1082 Tunis, Tunisie

\section{O. PLE}

Université Joseph-Fourier, 3SR, G-INP

CNRS UMR 5521

BP 53

38041 Grenoble Cedex 9,

France

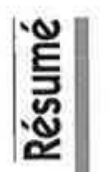

La liquéfaction des sols est un risque majeur pour les ouvrages construits ou fondés sur des terrains sableux et saturés. Létude proposée s'inscrit dans cette catégorie. Elle est appliquée à la fondation d'un barrage en terre dont le sol est formé essentiellement de couches de sables saturées. L'ouvrage étant situé dans une zone sismique, il convient donc de s'intéresser au comportement des sols de la fondation du barrage visà-vis du risque de liquéfaction. Ce risque était d'ailleurs à l'origine d'une opération de traitement de la fondation par vibrocompactage. Le présent article vise à évaluer le potentiel de liquéfaction de la fondation du barrage. Le travail porte sur l'exploitation des résultats des essais SPT (Standard Penetration Test) et CPT (Cone Penetration Test) effectués avant et après densification du sol par vibrocompactage. Les résultats obtenus ont permis de confirmer l'efficacité du vibrocompactage. Cette étude peut servir de base à d'autres analyses effectuées dans les mêmes conditions.

Mots-clés : sable lâche, liquefaction, SPT, CPT, vibrocompactage, barrage en terre.

\section{Evaluation of liquefaction risk of an earth dam foundation using in situ tests}

Soil liquefaction is a major risk for the design of earth structure built on sandy soils. This paper deals with the study of an earth dam foundation composed of saturated sand layers. Because the dam is located in a seismic area, the verification of liquefaction risk related to the dam and its foundation is recommended. To eliminate the potential risk of liquefaction especially for the soil foundation, a vibrocompaction treatment has been executed. In this paper, we present the interpretation of the Standard Penetration Test (SPT) and the Cone Penetration Test (CPT) results before and after soil densification. The exploitation of thesis results confirmed the efficiency of the vibrocompaction technique. This study can be used for other analysis realized in the same conditions.

Key words: loose sand, liquefaction, SPT, CPT, vibrocompaction, earth dam. 


\section{Introduction}

La liquéfaction des sables constitue l'un des phénomènes les plus importants et complexes de la dynamique des sols lâches. Dans des zones fortement sismiques, ce phénomène est responsable de dommages excessifs au niveau des fondations des ouvrages. Historiquement, les répercussions majeures, occasionnées en 1964 par le séisme de Nigata au Japon, ont stimulé plusieurs recherches pour appréhender le risque de liquéfaction et évaluer ses conséquences en fonction d'une intensité sismique donnée (Seed, 1971; Robertson \& Wride, 1998 ; Boulanger \& Idriss, 2008 ; Moss et al., 2006). Ainsi, plusieurs approches et critères empiriques ont été développés afin d'analyser le potentiel de liquéfaction d'un sol en se basant, en particulier, sur des résultats d'essais in situ.

Au Nord-Ouest de la Tunisie, le barrage Sidi El Barrak présente une fondation nettement envahies par des dépôts sableux perméables. A cet endroit, le risque sismique peut causer la liquéfaction des sables de l'ouvrage. C'est pourquoi, il est important de caractériser la susceptibilité du sol à la liquéfaction. Ainsi, le risque pourra être maitrisé et la fondation sera qualifiée. Cette étude explicitera, dans un premier temps par une présentation générale, la structure géologique et géotechnique de l'ouvrage de référence. Dans un deuxième temps, l'évaluation du potentiel de liquéfaction du sol sera effectuée à partir de l'analyse des résultats des essais in situ avant et après vibrocompactage. Enfin, un diagnostic de la fondation du barrage Sidi El Barrak sera présenté.

\section{Présentation du site}

Le barrage Sidi El Barrak est implanté sur l'oued Zouarâa à $15 \mathrm{~km}$ de la région de Nefza et à $20 \mathrm{~km}$ de la ville de Tabarka (Fig. 1). Le bassin versant couvre une superficie de $896 \mathrm{~km}^{2}$ et reçoit un apport annuel de l'ordre de 260 millions de $\mathrm{m}^{3}$ d'eau. En créant une réserve de 264 millions de $\mathrm{m}^{3}$, le barrage joue donc un rôle prépondérant dans la gestion des ressources en eau du pays.

Le barrage Sidi El Barrak est un ouvrage en terre caractérisé par une hauteur maximale de $28 \mathrm{~m}$ et une longueur en crête égale à $595 \mathrm{~m}$. Il s'agit d'une digue compactée et hétérogène dont la recharge amont est formée par un matériau argileux et la recharge aval est constituée par des sables provenant des fouilles de l'évacuateur de crues. De plus, il faut indiquer que dans le cas du barrage Sidi El Barrak, une paroi plastique a été mise en place afin de réduire les pertes par infiltration et d'éviter la manifestation des phénomènes d'érosion interne. Son ancrage a été fait dans le substratum marneux à une profondeur allant jusqu'à $34 \mathrm{~m}$ pour une épaisseur de $80 \mathrm{~cm}$.

\subsection{4}

\section{Contexte géologique du site}

La synthèse des résultats de la compagne géologique a permis de préciser la structure complexe de la fondation du barrage Sidi El Barrak. En effet, elle renferme des horizons perméables répartis aussi bien en surface qu'en profondeur et en contact direct avec d'autres niveaux de perméabilité plus faible. Ceuxci peuvent subir parfois une variation des faciès de l'amont vers l'aval et d'une rive à l'autre. D'une façon générale, les formations géologiques affleurantes se sont essentiellement formées au Paléogène, au Néogène et au Quaternaire. La rive gauche est constituée par des sables graveleux miopliocènes, de plusieurs dizaines de mètres d'épaisseur, reposant sur des terrains de l'oligocène. Ils sont recouverts par des sables dunaires éoliens plus au moins grésifiés et perméables. Le fond de la vallée est occupé par des terrains quaternaires recouverts par des sables alluvionnaires de 12 à $15 \mathrm{~m}$ de profondeur. A noter que l'ensemble de la fondation est subdivisé en sept groupes de terrains :
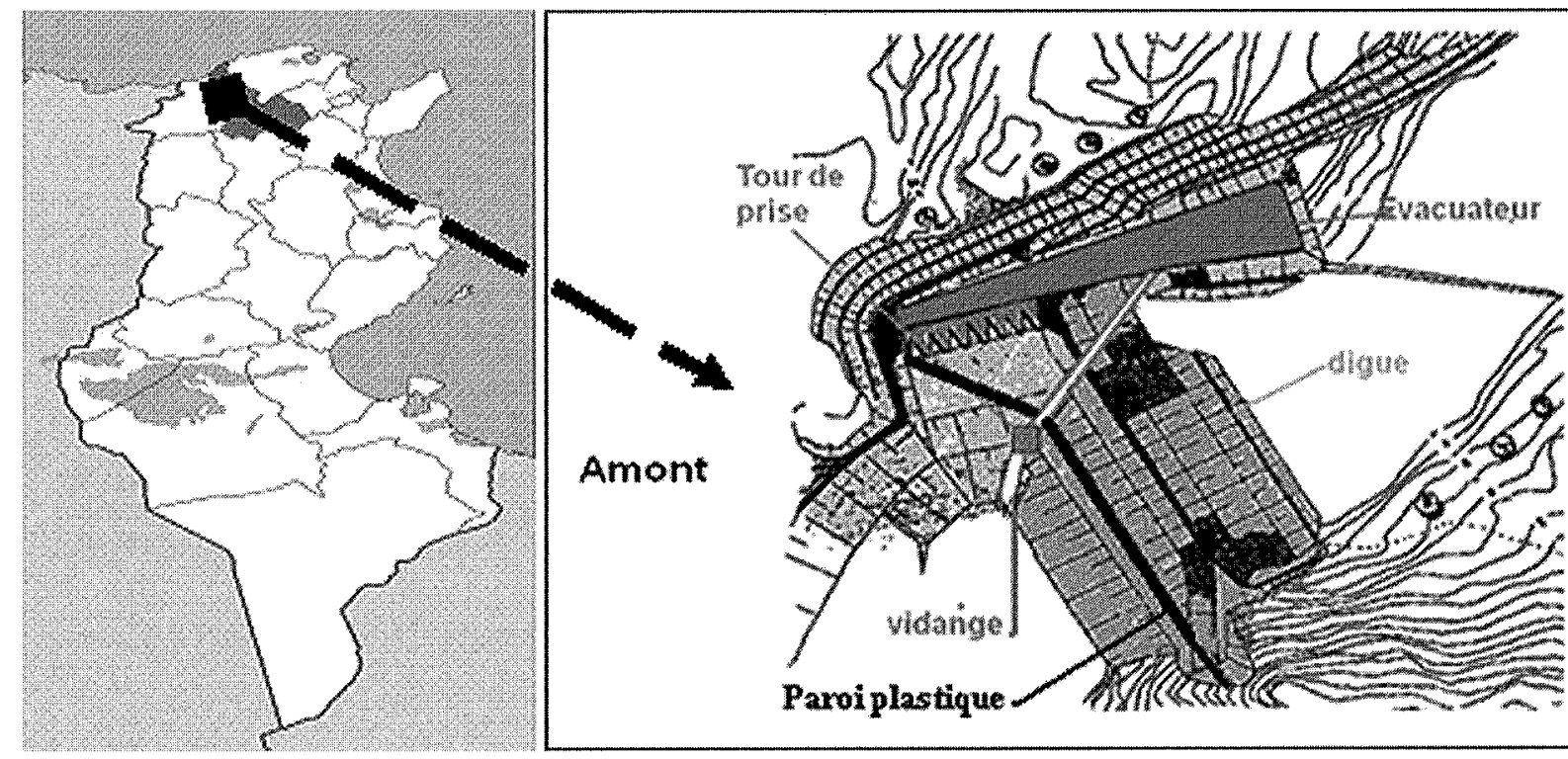

Aval

16. 1. Localisation du barrage Sidi El Barrak et description de ses composantes. Location of Sidi El Barrak dam and description of its components. 
- les terrains éoliens. Ils se présentent sous forme de sables fins à moyens, homogènes, à granulométrie serrée et de couleur jaune à brun. Leur épaisseur est importante au niveau de la rive gauche alors qu'elle est limitée à la rive droite surtout à l'aval de l'éperon rocheux ;

- les formations de pentes anciennes. Il s'agit de blocs de grés durs mélangés à des argiles vertes dont l'épaisseur peut être décimétrique à métrique. Les formations actuelles sont constituées de sables à débris de grès, de sables grossiers hétérogènes, de sables moyens homogènes de type éolien et de limons sableux à débris de grès ;

- les terrains alluvionnaires. Localisés dans le fond de la vallée, ces terrains sont constitués par des sables alluvionnaires homogènes, très uniformes et lenticulaires, à granulométrie moyenne, très serrée et de couleur jaune-beige jusqu'à $20 \mathrm{~m}$ de profondeur. Beaucoup plus en profondeur, il existe des terrains alluvionnaires hétérogènes formés par des lentilles des sables fins, de sables limoneux ou argileux et de graviers ;

- les terrains fluvio-lacustres. On les retrouve au niveau des horizons intermédiaires du fond de la vallée. Ainsi, on rencontre des argiles noires riches en matière organique, des sables limoneux, des limons ou des argiles sableuses de couleur grisâtre et quelques lentilles de sables fins ;

- les terrains dunaires du Quaternaire ancien. Placés à la rive gauche, ces terrains possèdent une structure classique de dunes éoliennes entrecroisées. Ils renferment parfois des dépressions dues à l'érosion ou à la dissolution. Ils comprennent des faciès sableux bruns beiges, des sables grésifiés et des bancs de grés friables ;

- les terrains miopliocènes continentaux. A la rive gauche, ils n'affleurent pas en surface et ils sont composés par des sables grésifiés, des sables moyens à fins, des sables limoneux conglomératiques et des argiles noires à brunes-vertes ;

- le substratum oligocène. Il renferme des marnes brunes-vertes et des bancs de grès siliceux.

Les figures 2 et 3 schématisent respectivement une coupe géologique transversale à l'oued et une coupe longitudinale de la fondation du barrage.

\section{2.}

\section{Contexte sismique}

Du point de vue tectonique, le site du barrage Sidi El Barrak est situé dans la zone de convergence des placues africaines et eurasiatiques de direction NNOSSE à NS. Il appartient à une zone de nappes de charriage où on distingue un axe de compression proche de l'horizontale et de direction N-S. Du point de vue historique, le foyer sismique le plus proche de la zone

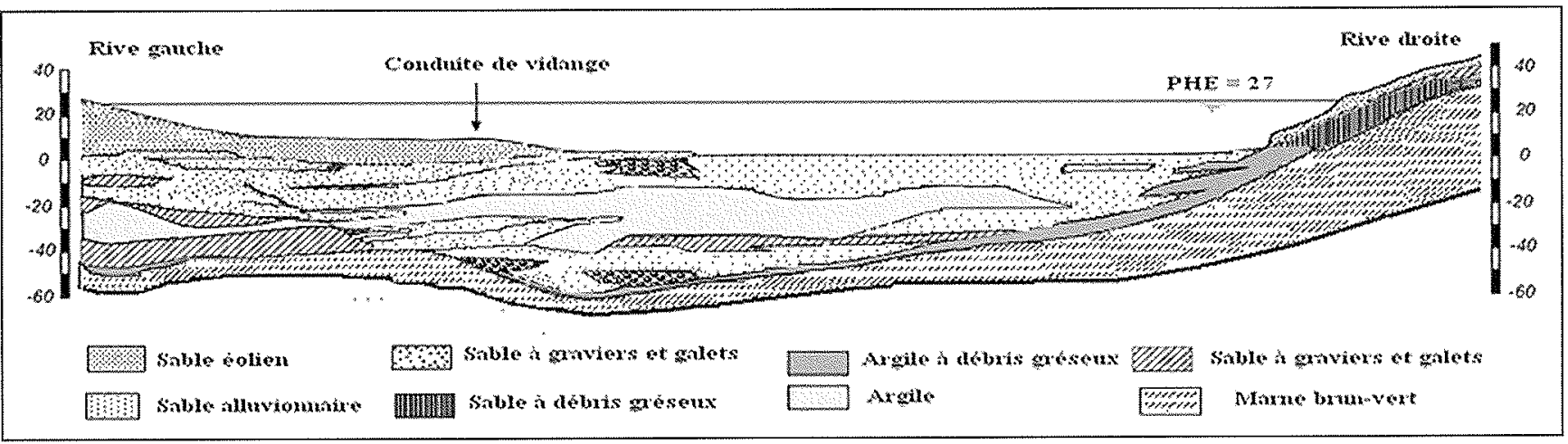

EG. 2 Coupe géologique longitudinale à l'oued.

Longitudinal section of the river.

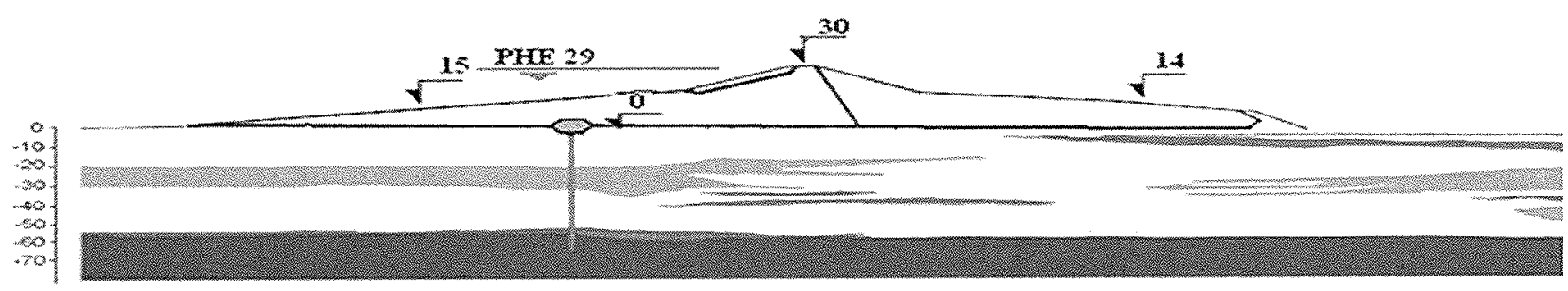

Le gende de la fondation

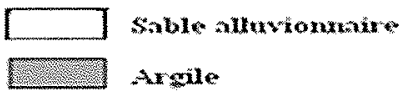

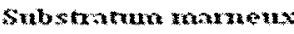

Snble abbrus sxestax

Argale

Blocs de gret dus enrobes ararale

Fic. 3 Coupe transversale de la fondation du barrage Cross section of the dam foundation. 
du barrage est localisé à l'est de Ghardimaou et, particulièrement, à l'intersection de la plaine de la moyenne de la Medjerda, d'une part (avec l'accident de Cap Serrât) et, d'autre part, avec les faisceaux des failles au sud de Sejnane et Nefza. Par ailleurs, la détermination des caractéristiques du séisme de référence est basée sur la méthodologie française d'évaluation du risque sismique pour les barrages. En effet, ces caractéristiques sont définies par une intensité épicentrale Io de VII MSK, une magnitude calculée égale à 5 et une profondeur du foyer de $10 \mathrm{~km}$. L'accélération maximale retenue pour le mouvement horizontal et le mouvement vertical vaut $0,15 \mathrm{~g}$ (Blanchin, et al., 2002).

\section{6}

\section{Contexte géotechnique}

Le site du barrage Sidi El Barrak a fait l'objet d'une campagne géotechnique comprenant des essais de laboratoire et des essais in situ. En effet, des puits de reconnaissance dans plusieurs zones ont été réalisés. A titre d'exemple, des puits ont été exécutés au niveau de la fondation : le premier puits a été réalisé dans la rive gauche (maille C2) où l'on a une prédominance des sables éoliens (Fig. 4a). Les autres puits ont été réalisés dans le lit de l'oued (mailles D4 et F3) où l'on a une abondance des sables alluvionnaires (Fig. 4b). La nappe de la rive gauche est à $5 \mathrm{~m}$ de profondeur, tandis que le lit de l'oued reste à un niveau superficiel. Ces résultats peuvent être exploités en vue de vérifier la susceptibilité des sables à la liquéfaction aux niveaux des deux zones (lit de l'oued et rive gauche). En effet, l'examen des conditions de liquéfaction des sables testés montre que le diamètre moyen $\mathrm{D}_{50}$ est compris entre 0,05 et 1,5 mm et le coefficient d'uniformité $\mathrm{C}_{\mathrm{U}}$ est inférieur à 15. Cela indique que le site du barrage Sidi El Barrak présente un potentiel de liquéfaction.

\section{3.}

\section{Traitement de la fondation par la technique du vibrocompactage}

La densification du sol du barrage Sidi El Barrak par la technique de vibrocompactage permet d'accroître la densité relative des horizons sableux à une valeur minimale de $70 \%$ alors que celle-ci, à l'état naturel, est souvent comprise entre 50 et $55 \%$. L'ensemble de la fondation est traité sur une profondeur allant jusqu'à $10 \mathrm{~m}$. La zone à densifier a été subdivisée en 45 éléments. A l'intérieur de chaque élément un maillage triangulaire est adopté pour le traitement avec des distances de 2,94 $\mathrm{m}$ et de $2,5 \mathrm{~m}$ entre les points de compactage (Fig. 5).

D'une façon générale, le vibreur est relié à une source d'énergie électrique et à une pompe à eau à haute pression (Fig. 5). Soumis aux fortes sollicitations du vibreur, le sol sableux saturé se liquéfie et le réarrangement des grains qui en résulte conduit à une compacité plus élevée.

Dans le cas du barrage Sidi El Barrak, le vibreur utilisé permet de compacter des alluvions jusqu'à une profondeur de $35 \mathrm{~m}$. Il a les caractéristiques suivantes : $0,355 \mathrm{~m}$ de longueur et une masse de $9500 \mathrm{~kg}$. Il est équipé d'un moteur de $120 \mathrm{~kW}$ qui impose une fréquence de $30 \mathrm{~Hz}$.

Par ailleurs, dans le but d'étudier l'efficacité de la technique de vibrocompactage, un suivi strict de la réaction du sol aux vibrations appliquées a été exécuté. En effet, plusieurs planches d'essais in situ (SPT et CPT) ont été réalisées avant et après vibrocompactage afin de quantifier les améliorations des caractéristiques du sol.

La figure 6 illustre des exemples de mailles, au niveau de la fondation du barrage Sidi El Barrak, traitées par la technique de vibrocompactage. Les résul-
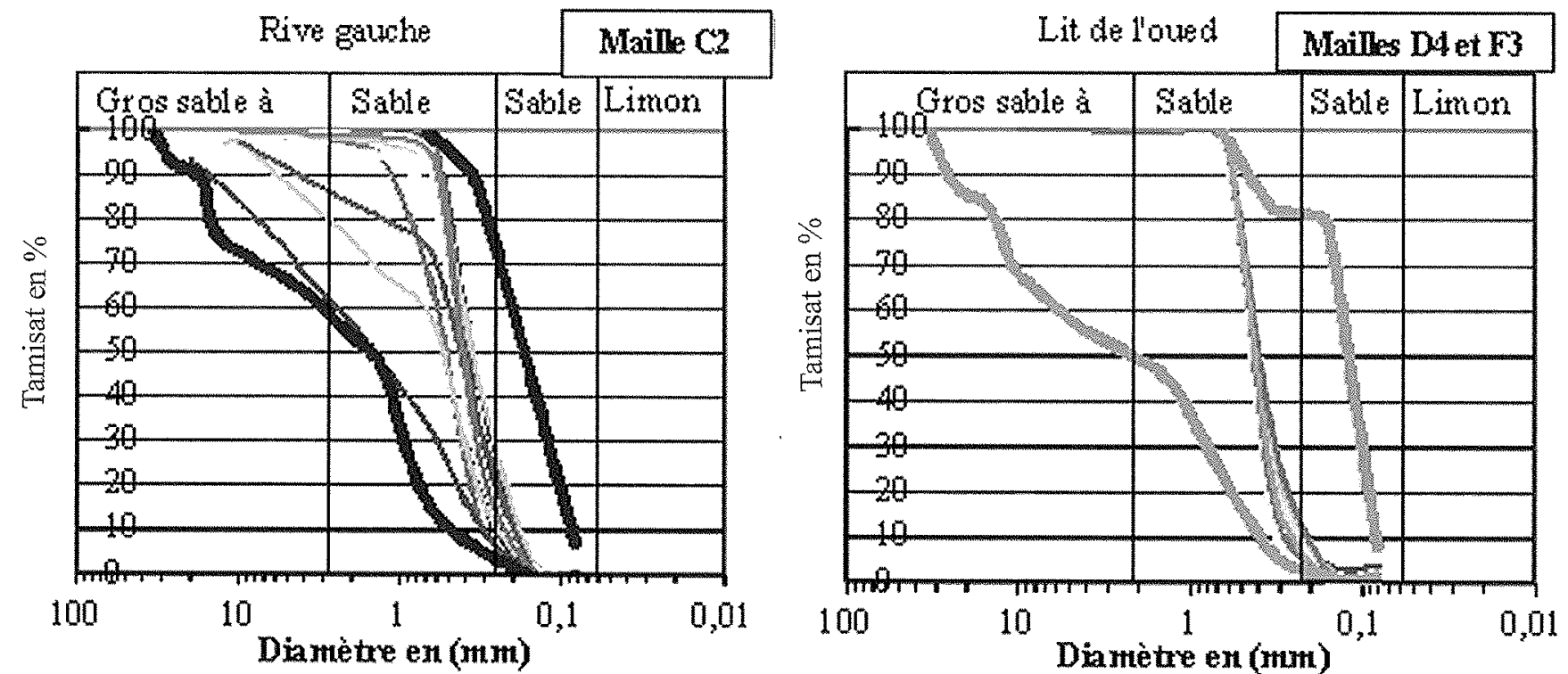

7G. 4 at $\mathrm{B}$ Fuseaux granulométriques des mailles C2, D4 et F3.

Grain distribution of soil in zones C2, D4 and F3. 

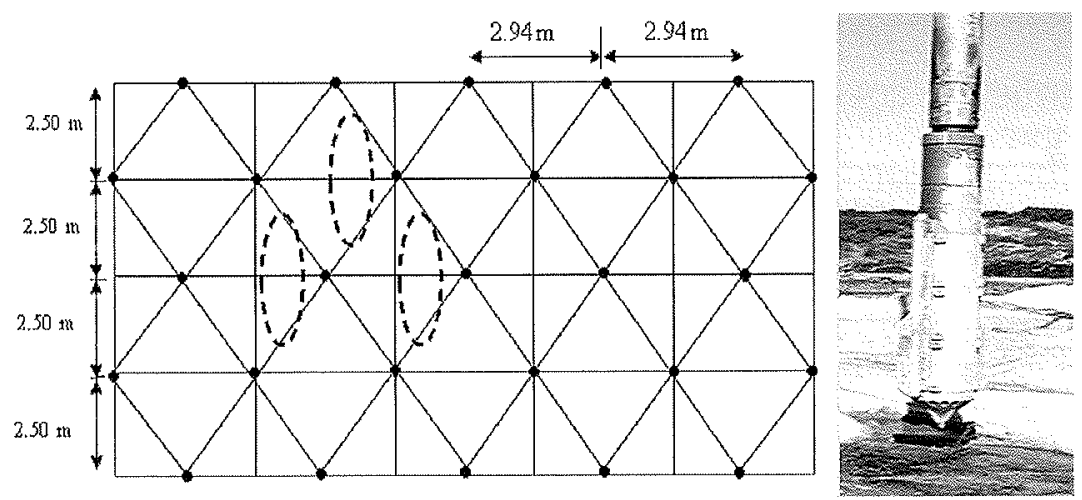

fF.s Maillage triangulaire traité par vibrocompactage et photo du vibrocompacteur.

Triangular mesh treated by vibrocompaction and the photo of the vibrocompactor.

tats des essais SPT sont disponibles dans les mailles désignées par C2, C4 et D4. La maille C2 est localisée à la rive gauche de l'ouvrage où on note une dominance des sables éoliens alors que les mailles C4 et D4 se situent au lit de l’oued qui est caractérisé par la présence des sables alluvionnaires. Identiquement aux essais SPT, pour évaluer le potentiel de liquéfaction des sols du barrage Sidi El Barrak, le traitement des résultats des essais CPT concerne les mailles suivantes : C2, C4 et F3. A noter que la maille F3 est située au lit de l'oued (Fig. 6).

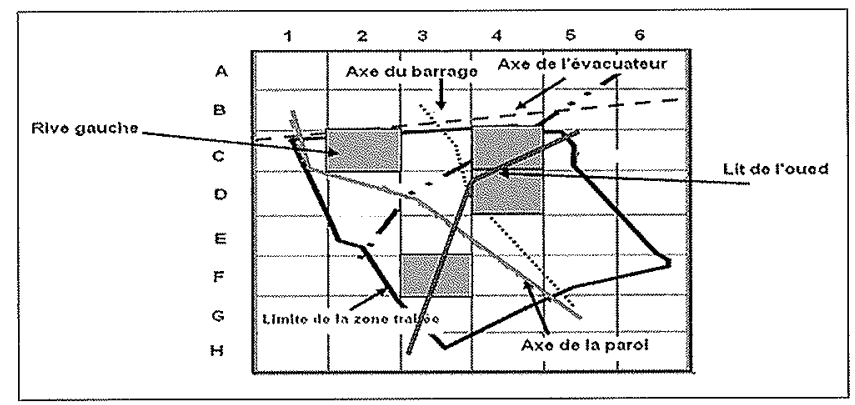

Exemples des mailles traitées par vibrocompactage (C2, C4, D4 et F3).

Examples of meshes treated by vibrocompaction (C2, C4, D4 and F3).

\section{3}

\section{Prévision du risque de liquéfaction à partir des essais in situ}

L'étude d'un ensemble de séismes aide à évaluer le potentiel de liquéfaction d'un sol. En effet, après le séisme de Nigata (1964), plusieurs chercheurs ont proposé de différencier les conditions de liquéfaction de celle de non liquéfaction. Pour cela, des investigations faites au moyen d'essais in situ ont permis de tirer les premiers critères empiriques de corrélation. Plus tard, en 1971, Seed et al. ont dégagé des corrélations qui tiennent compte de la contrainte effective et dans certaine mesure de la densité relative du sol étudié. Ces corrélations ont été présentées sous la forme de courbes enveloppes délimitant des domaines contenant des sites potentiellement liquéfiables. Par ailleurs, l'analyse de trente-cinq cas de sols liquéfiés au moment du séisme de Nigata (1964) montre que la contrainte de cisaillement moyenne peut être estimée à $65 \%$ de la contrainte de cisaillement maximale correspondant au pic de l'accélérogramme du séisme considéré (Seed, 1971). Ainsi, Seed a défini le rapport des contraintes cycliques par l'expression suivante :

$$
C S R=\frac{\tau_{a v}}{\sigma^{\prime} v}=0.65 \times \frac{\sigma_{v}}{\sigma^{\prime} v} \frac{\sigma_{\max }}{g} r_{d}
$$

où $\tau_{\mathrm{av}}$ est la contrainte de cisaillement moyenne due au séisme à la profondeur considérée, $\sigma_{v}^{\prime}$ est la contrainte verticale effective à la même profondeur, $\sigma_{v}$ est la contrainte verticale totale à cette profondeur, $\mathrm{a}_{\max }$ est l'accélération maximale du sol en surface $\left(\mathrm{m} / \mathrm{s}^{2}\right)$, g est l'accélération de la pesanteur $\left(\mathrm{m} / \mathrm{s}^{2}\right)$ et $\mathrm{r}_{\mathrm{d}}$ est un facteur correcteur de réduction de la contrainte de cisaillement avec la profondeur.

D’une manière générale, l'évaluation du risque de liquéfaction est fondée sur la détermination de la résistance du sol et de sa comparaison au rapport des contraintes cycliques généré par le séisme. Elle permet d'évaluer la sécurité des sols contre le phénomène de liquéfaction en utilisant l'expression suivante :

$$
F S=\frac{C R R}{C S R} *
$$

avec CRR (Cyclic Resistance Ration) la résistance du sol à la liquéfaction qui est déterminée empiriquement. Elle représente la courbe limite dans le plan (contraintes sismiques, résistance in situ) entre les sites liquéfiés et ceux non liquéfiés. CSR (Cyclic Stress Ratio) qui est le rapport des contraintes cyclique calculé par l'équation 1. MSF (Magnitude Scaling Factor) qui est le facteur d'échelle d'amplitude. L'analyse du potentiel de liquéfaction est faite au moyen des investigations réalisées post tremblement de terre d'amplitude de référence 7,5. Le coefficient MSF a donc été déterminé pour obtenir une valeur corrigée de CSR correspondant à une magnitude égale à 5 .

Parmi les relations empiriques bien connues, il faut mentionner celle de Boulanger et Idriss (2008) dont l'objectif principal consiste à calculer la résistance cyclique d'un sol (CRR) à partir des résultats des essais SPT. Également, les méthodes de Robertson et al. (1998) et celle de Moss et al. (2006) permettent de calculer CRR en utilisant les données des essais CPT. 


\section{sing}

\section{Évaluation du potentiel de liquéfaction à partir des essais SPT}

Dans un essai SPT, il est nécessaire de corriger la résistance à la pénétration en fonction du nombre de coups compté. En effet, comme la résistance du sol et son comportement vis-à-vis du risque de liquéfaction sont contrôlés par l'état des contraintes effectives, une correction avec la profondeur est nécessaire. Le rapport des contraintes cycliques peut être exprimé en fonction d'une valeur corrigée de la résistance à la pénétration $\mathrm{N}_{1}$ (Corté, 1978). La relation entre la résistance à la pénétration mesurée $\mathrm{N}$ et $\mathrm{N}_{1}$ est donnée par l'équation suivante :

$$
N_{1}=C_{N}^{*}
$$

Avec $\mathrm{N}$ la résistance à la pénétration mesurée, $\mathrm{N}$, la résistance à la pénétration corrigée et $C_{N}$ le coefficient multiplicateur qui dépend de la densité relative, de la contrainte effective et la résistance de pénétration mesurée.

Les figures 7 et 8 représentent la résistance à la pénétration corrigée $N_{1}$ en fonction de la profondeur. Ces figures se basent sur les résultats des essais SPT effectués avant et après vibrocompactage pour les mailles C2, C4 et D4. Ces résultats indiquent une nette augmentation des valeurs de $\mathrm{N}_{1}$ obtenues après vibrocompactage En effet, la valeur moyenne de $\mathrm{N}_{1}$ des horizons non traités est de l'ordre de 21, 13 et 8 coups par 0,3 m (Fig. 7) respectivement pour les mailles C2, C4 et D4 alors qu'elle atteint 46, 39 et 40 coups par $0,3 \mathrm{~m}$ pour les couches densifiées (Figs. 10, 11 et 12).

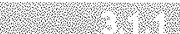

\section{Méthode de Boulanger et Idriss (2008)}

Cette relation consiste à déterminer la résistance du sol à la liquéfaction en se basant sur des valeurs modifiées de la résistance à la pénétration mesurée. Boulanger et Idriss (2008) ont construit des courbes enveloppes pour des sols non cimentés, d'âge holocène et à différents pourcentages de fines. Ces courbes sont utiles pour l'identification des sites liquéfiables. L'équation 4 décrit la relation entre la résistance cyclique et la résistance corrigée (Boulanger et al., 2008).

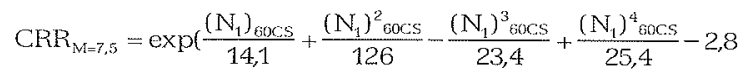

avec $\left(N_{1}\right)_{\text {6ocs }}$ la résistance équivalente à celle d'un sable propre dont la détermination s'effectue en employant les expressions donnée par les équations suivantes :

$$
\begin{gathered}
N_{1,60}=N \cdot C_{N} \cdot C_{S} \cdot C_{R} \cdot C_{B} \cdot C_{E} \\
\left(N_{1}\right)_{60 \mathrm{cs}}=N_{1,60}+\Delta\left(N_{1,60}\right) \\
\Delta(N 1,60)=\exp \left(1,63+\frac{9.7}{F C+0.01}-\left(\frac{15.7}{F C+0.01}\right)^{2}\right)
\end{gathered}
$$

avec, $\mathrm{N}$ le nombre de coups mesuré (coups par $0,3 \mathrm{~m}$ ), $\mathrm{C}_{\mathrm{N}}$ la correction de la contrainte effective du sol, $\mathrm{C}_{\mathrm{E}}$ la correction du taux d'énergie de la masse, $\mathrm{C}_{\mathrm{B}}$ la Cor- $^{-}$ rection du diamètre de forage, $\mathrm{C}_{\mathrm{B}}$ la correction de la longueur du train des tiges, $\mathrm{C}_{\mathrm{S}}$ la correction du carottier avec ou sans revêtement et $\mathrm{FC}$ le pourcentage des fines.
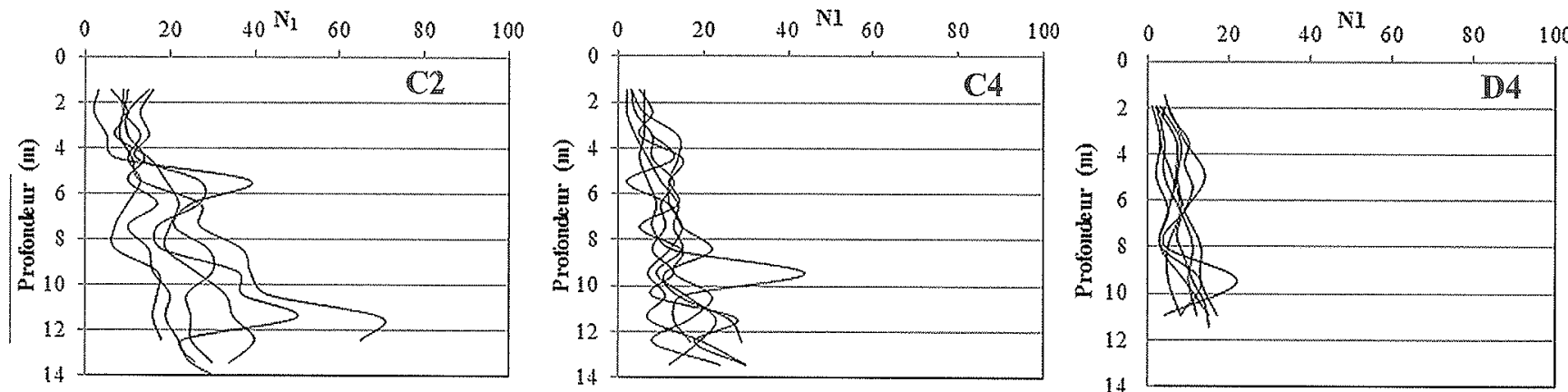

FG. 7 Résultats des essais SPT dans la maille C2, C4 et D4 avant vibrocompactage.

SPT results in mesh C2, C4, D4 before vibrocompaction.
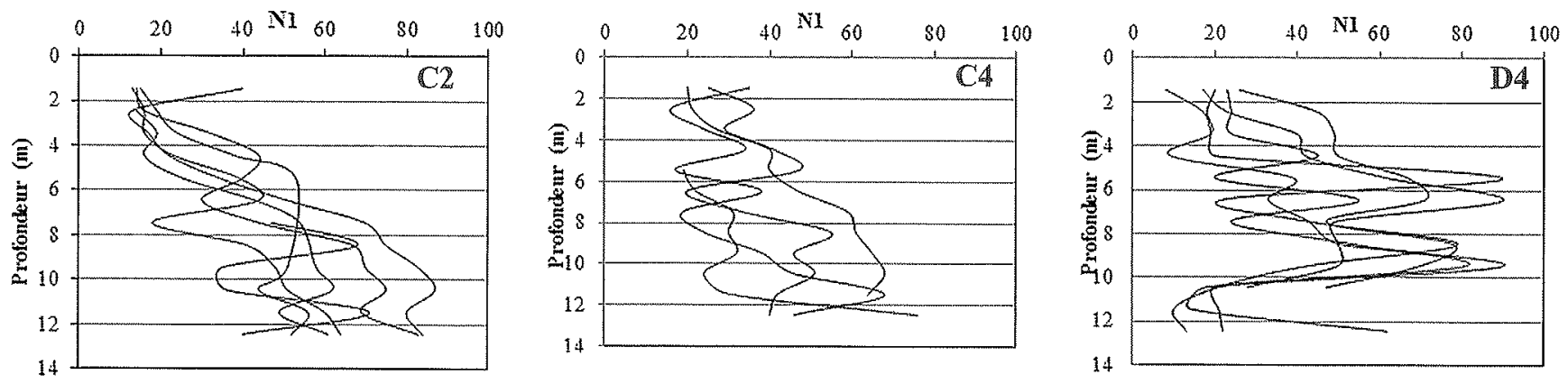

Fic. 8 Résultats des essais SPT dans la maille C2, C4 et D4 après vibrocompactage SPT results in mesh C2, C4, D4 after vibrocompaction. 
La figure 9 illustre le rapport des contraintes cycliques (CSR) en fonction de la résistance à la pénétration corrigée enregistrée avant et après vibrocompactage (mailles C2, C4 et D4). Avant le traitement de la fondation, il est clair que les points (points foncés) sont situés au-dessous de la courbe enveloppe séparant les sites liquéfiables de ceux non liquéfiables. Après vibrocompactage, le nuage des points (points clairs) est totalement décalé à droite de la courbe enveloppe, c'est-àdire dans le domaine non liquéfiable. En conclusion, les formations sableuses compactées ne présentent pas de risque de liquéfaction. De plus, à chaque point de la fondation, on peut former le rapport entre la résistance à la liquéfaction et la sollicitation cyclique, appelé encore facteur de sécurité dont la valeur oscille autour de 1 suivant l'occurrence $(F S<1)$ ou l'absence $(F S>1)$ de risque de liquéfaction. La représentation de ce paramètre se fait sous forme de graphiques ou de cartes. La figure 10 indique la variation du facteur de sécurité calculé avant vibrocompactage dans chacune des trois mailles de la fondation du barrage Sidi El Barrak.

D'après cette figure, on note que le facteur de sécurité est faible et ne dépasse pas la valeur de 1 . En effet, la valeur moyenne est comprise entre 0,7 et 0,85 dans les mailles C2 et C4 alors qu'elle varie de 0,6 à 0,9 dans la maille D4. Ces résultats confirment bien la tendance de la fondation à se liquéfier. Une consolidation du sol est donc raisonnable. La figure 10 montre les profils de facteur de sécurité des horizons densifiés par vibrocompactage dans les mêmes endroits (mailles C2, C4 et D4). D'après les résultats obtenus, on note que les valeurs du facteur de sécurité (FS) sont, maintenant, plus élevées et nettement supérieures à 1. Ceci indique que le sol a acquis une résistance suffisante pour minimiser le risque de liquéfaction vis-à-vis d’un séisme.

\section{5}

\section{Évaluation du potentiel de liquéfaction à partir des essais CPT}

On rappelle que dans la présente étude, l'examen des résultats des essais CPT intéressera les mailles C2, C4 et F3. L'analyse des données des essais CPT effectués avant et après vibrocompactage (Figs. 12 à 13) montre que l'effet de densification est nettement meilleur dans les trois mailles C2, C4 et F3. Cette densification est décrite par une augmentation des valeurs de $q_{c}$ enregistrées après vibrocompactage par rapport à celles notées avant le traitement de la fondation. Dans le cas du sol traité, les valeurs moyennes de $q_{c}$ dépassent $15 \mathrm{MPa}$, tandis qu'elles sont inférieures ă $12 \mathrm{MPa}$ pour le sol non traité.

\section{-}

\section{Méthode de Robertson et Wride (1998)}

Dans le but d'apprécier le potentiel de liquéfaction des sols sableux, les approches empiriques basées sur les essais CPT nécessitent la connaissance préalable de certaines caractéristiques du sol, en particulier, le pourcentage des fines ou le diamètre moyen $D_{50}$ ou bien encore la connaissance des corrélations SPT-CPT. Robertson et Wride (1998) ont introduit une nouvelle procédure pour évaluer le potentiel de liquéfaction en se basant uniquement sur les données issues des essais CPT. Cette méthode s'appuie sur la résistance de pointe statique $q_{c}$ et la mesure du frottement latéral sur le manchon $\mathrm{f}_{\mathrm{s}}$. Le principe consiste à déterminer la résistance d'un sol à la liquéfaction en faisant référence à un sable propre. Pour cette raison, la résistance de pointe mesurée $q_{c}$ est, en premier lieu, normalisée en une résistance $q_{c 1 N}$ puis corrigée pour obtenir une valeur équivalente $q_{\mathrm{c} 1 \mathrm{~N}) \mathrm{cs}}$ relative à un sable propre (Robertson et al., 1998). Enfin, en fonction des valeurs de référence, $\mathrm{q}_{(1 \mathrm{~N}) \mathrm{s} \mathbf{s}^{\prime}}$ on obtient une formulation de la résistance à la liquéfaction CRR qui est décrite par les équations suivantes (Robertson et al., 2000) :

- pour $\mathrm{q}_{\mathrm{c} 1 \mathrm{~N}) \mathrm{cs}}<50 \mathrm{CRP}_{7,5}=0,833\left[\left(\mathrm{q}_{\mathrm{c} 1 \mathrm{~N}) \mathrm{cs}}\right) / 1000\right]+0,05$

- pour $50<\mathrm{q}_{\mathrm{c} 1 \mathrm{n}) \mathrm{cs}}<160 \mathrm{CRR}_{7,5}=93\left[\left(\mathrm{q}_{\mathrm{c} \text { nn)s }}\right) / 1000\right]^{3}+0,08$

Généralement, les relations de la normalisation présentées par Robertson et Wride (1998) s'expriment par les équations suivantes:

$$
\begin{gathered}
\mathrm{q}_{\mathrm{c} 1 \mathrm{~N}}=\mathrm{C}_{\mathrm{q}}\left[\mathrm{q}_{\mathrm{c}} / \mathrm{P}_{\mathrm{a} 1 \mathrm{1}}\right] \\
\mathrm{C}_{\mathrm{q}}=\left[\mathrm{P}_{\mathrm{a} 2} / \sigma_{\mathrm{v}}^{\prime}\right]^{\mathrm{n}} \\
\mathrm{F}=\left[\mathrm{f}_{\mathrm{s}} /\left(\mathrm{q}_{\mathrm{c}}-\sigma_{\mathrm{v}}\right)\right]^{*} 100 \%
\end{gathered}
$$

$q_{c 1 N}$ est la résistance de pointe normalisée, $q_{c}$ la résistance de pointe mesurée $(\mathrm{MPa}), \mathrm{C}_{\mathrm{q}}$ le coefficient correcteur dont une valeur maximale égale à 2 est généralement recommandée dans le cas des faibles profondeurs, $\sigma^{\prime}$ la contrainte verticale effective (kPa), $\sigma_{\mathrm{v}}$ la contrainte verticale totale $(\mathrm{kPa}) ; \mathrm{P}_{\mathrm{a} 1}$ et $\mathrm{P}_{\mathrm{a} 2}$ les pressions de références ayant des unités respectivement identiques à celles de $q_{c}$ et $\sigma_{V}^{\prime}\left(P_{a 1}=100 \mathrm{kPa}\right.$ si $\sigma^{\prime}{ }_{v}$ est exprimée en $\mathrm{kPa}, \mathrm{P}_{a 2}=0,1 \mathrm{MPa}$ si $\mathrm{q}_{c}$ est mesurée en $\mathrm{MPa}, \mathrm{n}$ l'exposant des contraintes variant de 0,5 pour un sable propre jusqu'à 1 pour un sol argileux, F le frottement normalisé et $\mathrm{f}_{\mathrm{s}}$ le frottement latéral, exprimé en $\mathrm{kPa}$.
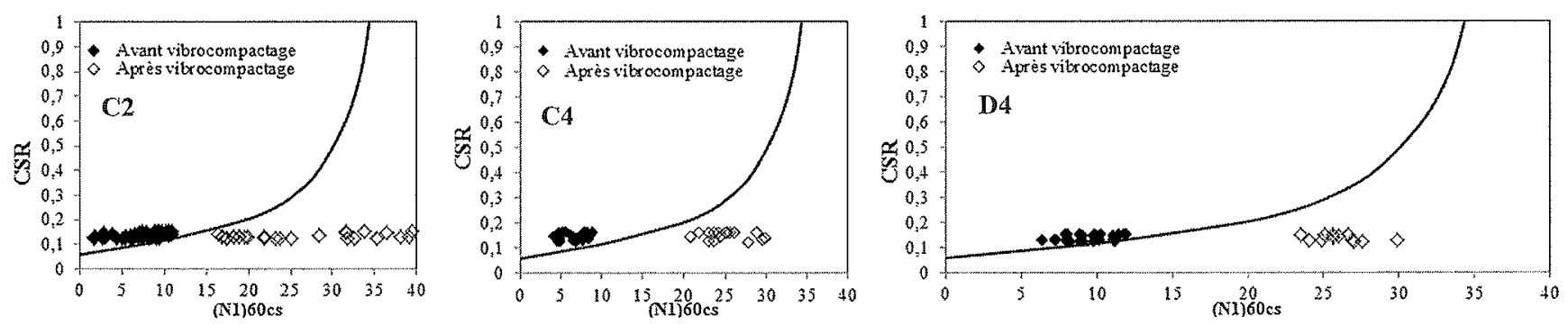

16. 9 CSR en fonction de $\left(N_{1}\right)_{60, s}$ dans $\mathrm{C} 2, \mathrm{C} 4$ et $\mathrm{D} 4$ CSR as a function of $\left(\mathrm{N}_{1}\right)_{60 \mathrm{cs}}$ in $\mathrm{C} 2, \mathrm{C} 4, \mathrm{D} 4$. 


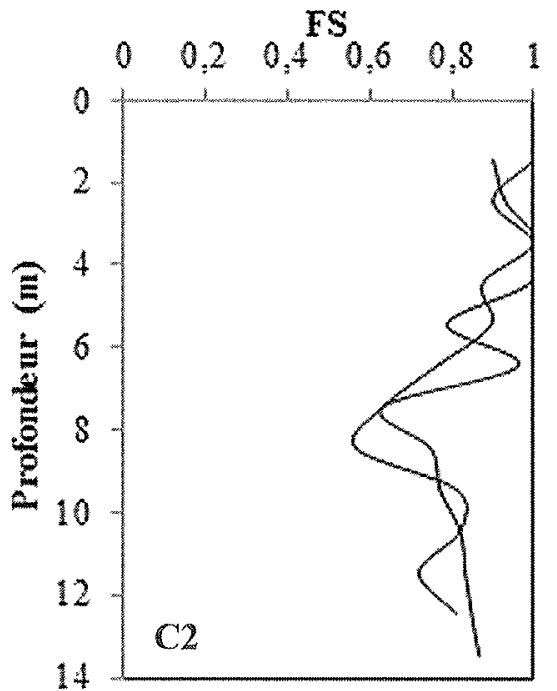

FS

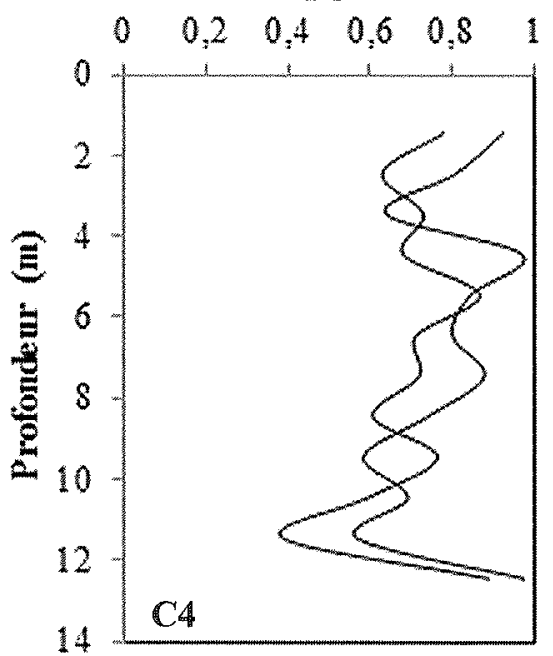

FS

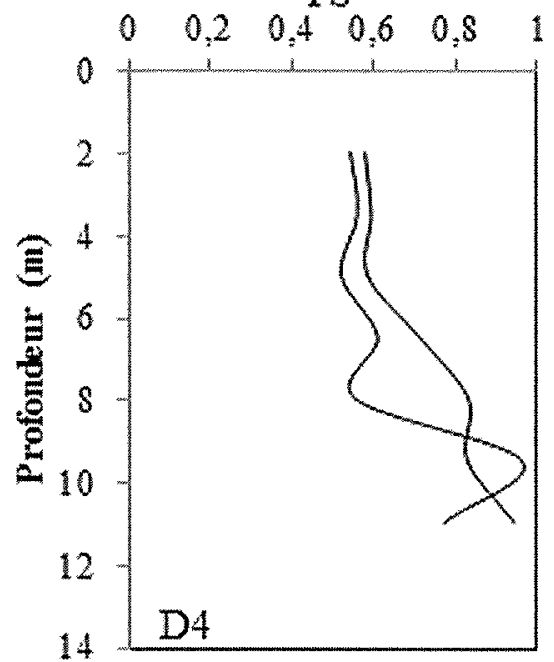

16. to Variation de FS avant vibrocompactage dans les mailles C2, C4 et D4. Variation of FS before vibrocompaction in meshes C2, C4 and D4.

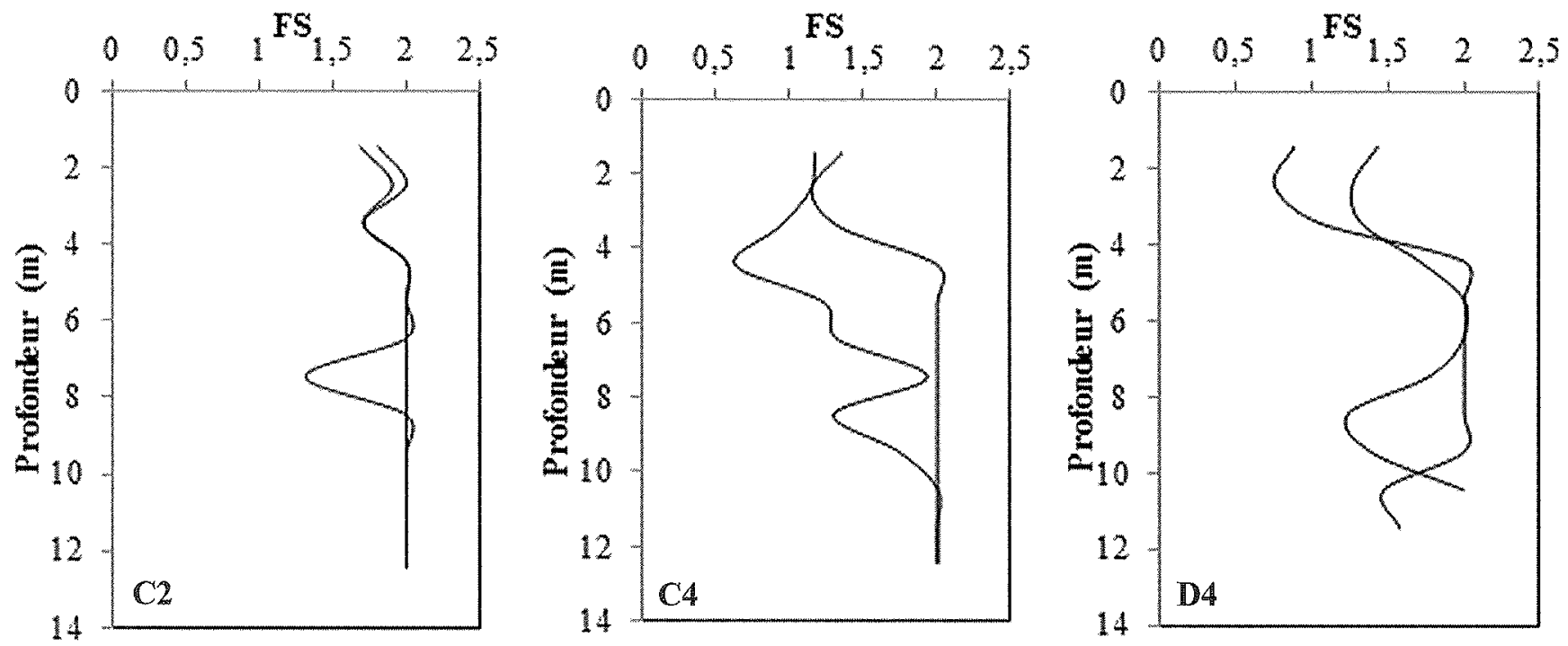

16. 1 t Variation de FS après vibrocompactage dans les mailles C2, C4 et D4. Variation of FS after vibrocompaction in meshes C2, C4 and D4.
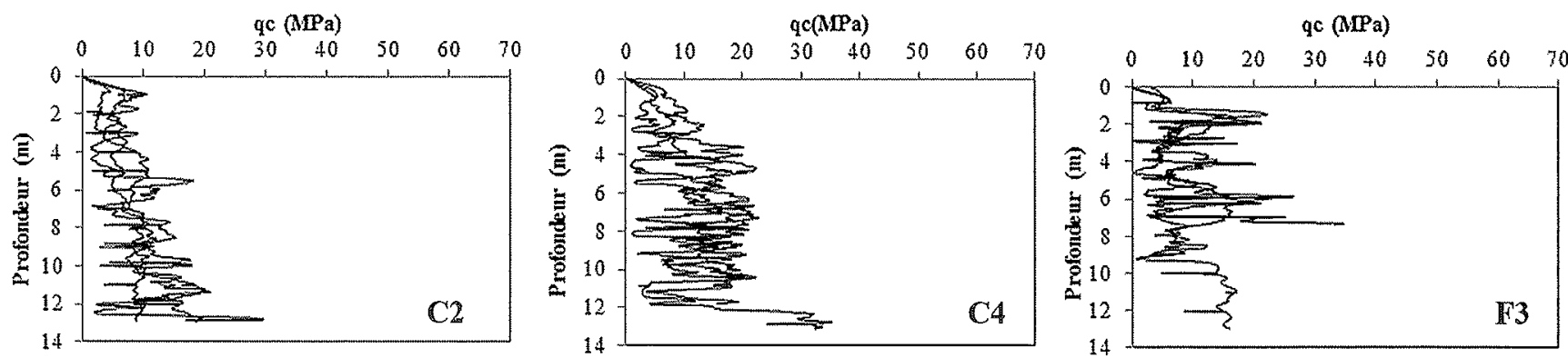

16. 10 Résultats CPT de C2, C4, F3 avant traitement. CPT results of C2, C4, F3 before treatment. 

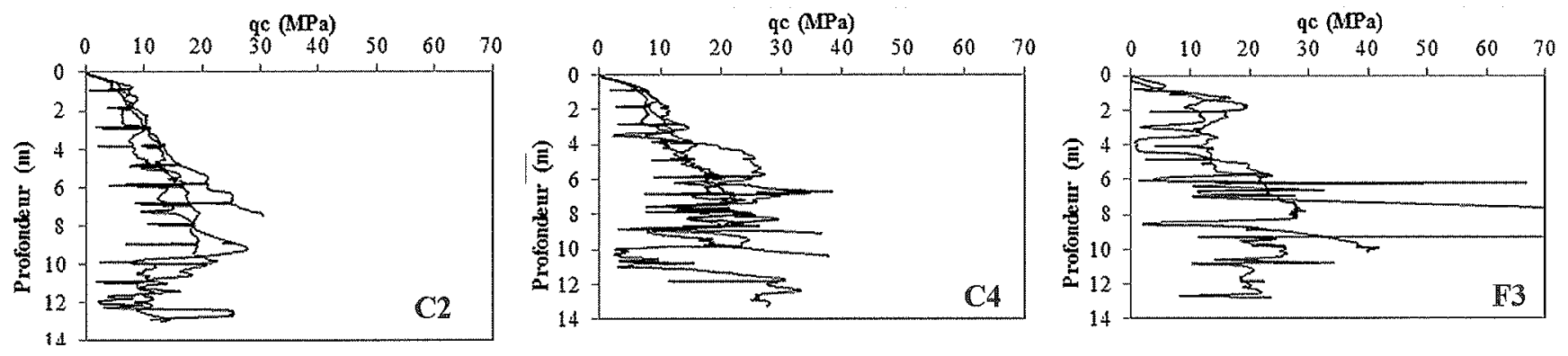

16. 18 Résultats CPT de C2, C4, F3 après traitement.

CPT results of $\mathrm{C} 2, \mathrm{C} 4, \mathrm{~F} 3$ after treatment.

\section{Mencis}

\section{Classification des sols en essai CPT}

L'avantage majeur que présente un essai CPT par rapport à un essai SPT consiste à reconnaitre directement le profil stratigraphique et la nature du terrain traversé puisqu'il fournit une lecture continue avec la profondeur. A cet effet, Robertson et Wride (1998) proposent de déterminer les caractéristiques des particules du sol au moyen d'un abaque, représentant le comportement des couches rencontrées, où figurent des matériaux de différentes natures. Sur cet abaque, les frontières séparant les zones indiquées (2 à 7) peuvent être rapprochées à des cercles concentriques. Le rayon de chaque cercle est utilisé comme un indice de comportement I dont la valeur est globalement utile pour identifier les propriétés du sol. Cet indice est défini par l'équation suivante:

$$
I_{c}=\left[(3,47-\log Q)^{2}+(1,22+\log F)^{2}\right]^{0,5}
$$
avec, $I_{c}$ l'indice de comportement du sol, $Q$ la résistance de pointe normalisée et $F$ le frottement latéral normalisé.

Le tableau I explicite les différents comportements du sol en fonction des valeurs de l'indice $I_{c}$

La figure 14 montre les différents types de sols des mailles C2, C4 et F3 selon l'abaque de Robertson et Wride (1998). D'après les courbes tracées, on peut dégager les constatations suivantes:

- à la rive gauche (Fig. 14a) le nuage des points, au niveau de la maille $\mathrm{C} 2$, est essentiellement situé dans les zones 6 et 7 ce qui indique que les couches du sol de la fondation ont un comportement semblable à celui d'un sable propre à silteux dans la première zone et à celui d'un sable graveleux dans la deuxième zone (tableau I). Ceci est en accord avec les résultats de la

TABtesu I Relation entre $I_{c}$ et le type du sol (Robertson et al., 2000).

\begin{tabular}{c|c|c|}
\hline & Wubed sol \\
\hline$I_{c}<1,31$ & 7 & Sable gravier à sable dense \\
$1,31<I_{c}<2,05$ & 6 & Sable propre à sable silteux \\
$2,05<I_{c}<2,60$ & 5 & Sable mélangé \\
$2,60<I_{c}<2,95$ & 4 & Argile et silt \\
$2,95<I_{c}<3,60$ & 3 & Argile silteuse à argile pure \\
\hline
\end{tabular}
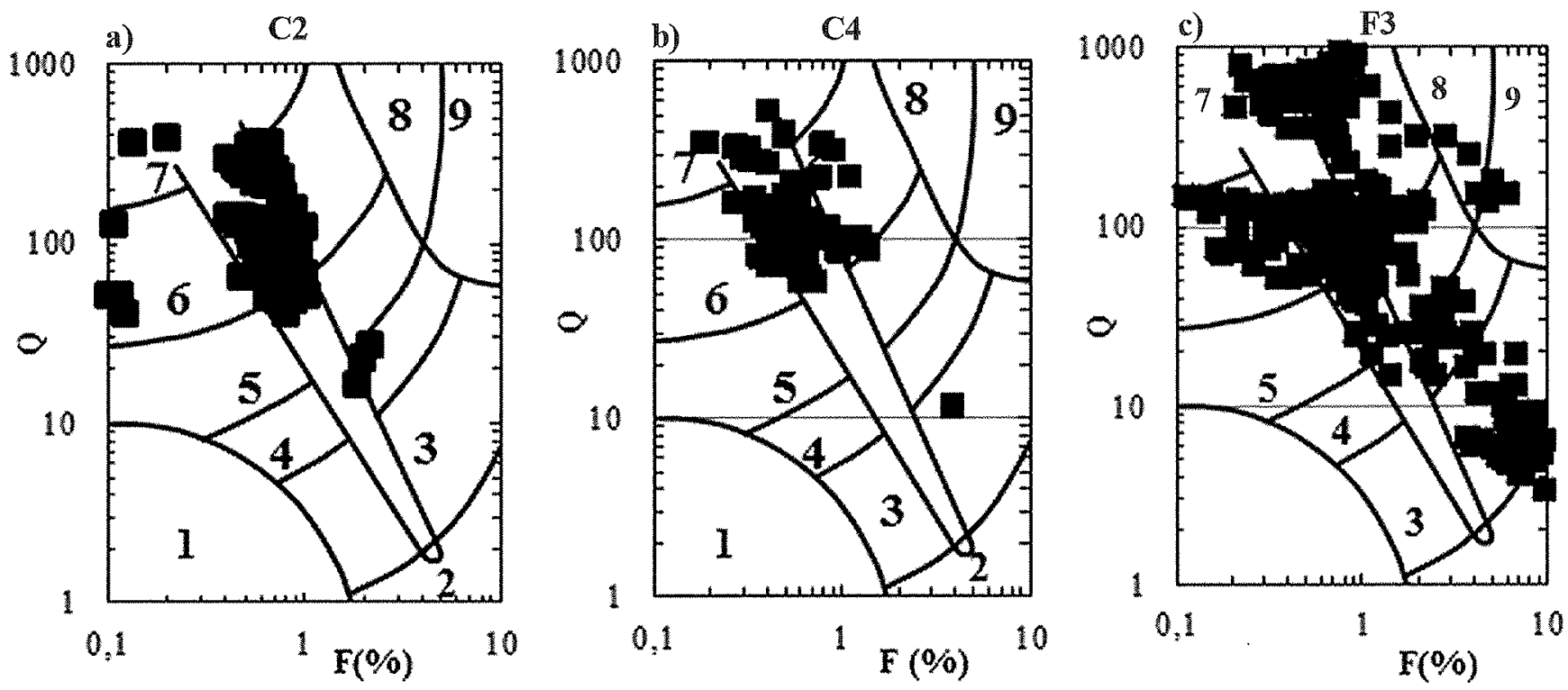

16. 14 Classification des sols de $\mathrm{C} 2, \mathrm{C} 4, \mathrm{~F} 3$. Classification of soils of $\mathrm{C} 2, \mathrm{C} 4, \mathrm{~F} 3$. 
reconnaissance géologique qui prouve que la rive gauche est occupée par des sables graveleux hétérogènes à passées limoneuses, surmontés par des sables éoliens de plusieurs dizaines de mètres d'épaisseur ;

- au lit de l'oued, au niveau des mailles C4 et F3, les points sont repartis sur les zones $2,3,4,5,6,7,8,9 \mathrm{ce}$ qui montre la présence d'une grande variété des matériaux allant des argiles silteuses aux sables graveleux (tableau I, fig. 14b-c). Ces résultats sont aussi en bonne concordance avec l'étude géologique qui affirme que le fonds de la vallée renferme des sédiments argileux, des alluvions silteuses et principalement des sables alluvionnaires.

\section{a}

\section{Détermination de la résistance équivalente d'un sable propre en essai CPT}

D’après Robertson et Wride (1998), il est nécessaire de corriger la résistance de pointe afin d'amélioration la résistance à la liquéfaction sous l'effet d'un accroissement du pourcentage des fines. L'approche de Robertson et Wride (1998) fait donc appel à une distinction entre les horizons granulaires, pour lesquels ce genre de correction est envisageable, et ceux à comportement argileux pour lesquels certains critères doivent être vérifiés. Parmi ces critères on distingue le critère chinois (Youd et al., 1997) qui se base sur l'indice de comportement $I_{c}$, calculé pour $n=1$ (Fig. 15). Les couches identifiées comme argileuses sont situées au-delà de la droite limite correspondant à un indice $I_{c}$ égal à 2,6. L'allure de chaque courbe permet de constater que :

- dans la zone de la rive gauche, l'examen du profil présenté par la figure 15a conduit à mettre en évidence, pour la maille $\mathrm{C} 2$, deux niveaux à comportement argileux : le premier s'étend jusqu'à 1,15 m par rapport à la surface du sol et le deuxième est localisé à $3 \mathrm{~m}$ de profondeur;

- dans la zone du lit de l'oued, concernant la maille C4, la figure $15 \mathrm{~b}$ montre des oscillations occasionnelles de l'indice de comportement $I_{c}$ par rapport à sa valeur limite $\left(I_{c}=2,6\right)$ indiquant ainsi une alternance nette entre des couches fines ou argileuses et des couches sableuses :
- d'après la figure 15c, il apparaît que, pour la maille F3, le comportement argileux peut être assigné aux horizons situés dans les trois premiers mètres et à ceux qui se trouvent entre 8,5 et $11 \mathrm{~m}$ de profondeur.

Ce critère montre que le potentiel de liquéfaction cyclique dans les limons et les argiles est souvent gouverné par les dimensions des particules du sol, la limite de liquidité et la teneur en eau. En effet, le critère chinois stipule que la liquéfaction ne pourra se produire que si les conditions suivantes sont réunies (Youd et al., 1997).

1) Le pourcentage d'argile présente dans le sol est inférieur à $15 \%$. 2) La limite de liquidité $W_{\mathrm{I}}$ est inférieure à $35 \%$. 3) La teneur en eau ne dépasse pas $90 \%$ de la limite de liquidité.

Les tableaux II et III présentent les différentes caractéristiques des sols avant vibrocompactage pour des échantillons prélevés de la rive gauche et du lit de l'oued. D’après le tableau 2, les résultats d'identification des sols en laboratoire montrent que le critère chinois (Youd et al., 1997) n'est pas vérifié puisque la teneur en argile dépasse $15 \%$ et la limite de liquidité est très nettement supérieure à $35 \%$. Il en ressort donc que les couches argileuses de la maille C2 ne sont pas sensibles à la liquéfaction. D'après le tableau 3, il en va de même pour les mailles C4 et F3.

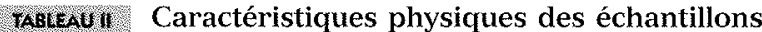
argileux prélevés de la maille $\mathrm{C} 2$.

Characteristics of clayey samples of the mesh C2.

\begin{tabular}{|c|c|c|c|}
\hline Mane & $\begin{array}{l}\text { White } \\
\text { ate liturde }\end{array}$ & $\begin{array}{l}\text { Terreu } \\
\text { carre }(0)\end{array}$ & 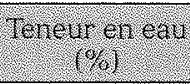 \\
\hline C2 & 78 & 54,1 & 56 \\
\hline
\end{tabular}

TABIEuin Caractéristiques physiques des échantillons argileux des deux mailles C4 et F3. Characteristics of clayey samples of the meshes $\mathrm{C} 4$ and F3.

\begin{tabular}{|c|c|c|c|}
\hline Malle & 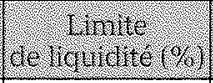 & $\begin{array}{l}\text { Teneur } \\
\text { enarguc ror }\end{array}$ & 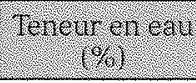 \\
\hline $\mathrm{C} 4$ & 53 & 34,7 & 21,5 \\
\hline F3 & 44 & 34 & 18,7 \\
\hline
\end{tabular}
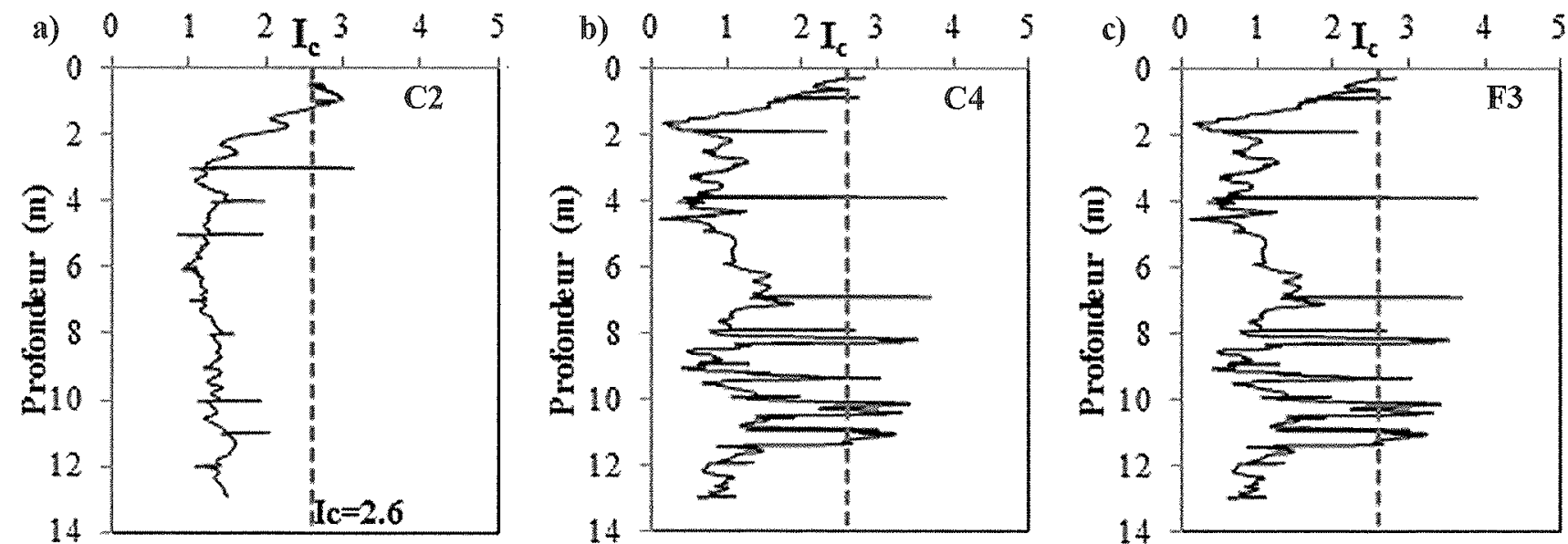
Selon Robertson et al. (1998), si l'indice de comportement du sol est minimum par rapport à sa valeur limite, le sol est classé comme granulaire et un calcul de I s'avère nécessaire avec un changement de l'exposant $n$ pour juger si les couches granulaires sont plastiques ou non plastiques. Dans ce cas, des profils de l'indice de comportement $I_{c}$ ont été tracés, pour n égal à 0,5 afin de caractériser la plasticité des couches. La figure 16 décrit les profils établis de l'indice de comportement des terrains de chaque maille de la fondation du barrage. D'après ces figures, on constate que, sur la totalité des mailles étudiées, l'indice de comportement $I_{c}$ est inférieur à 2,6 ce qui signifie qu'il $s^{\prime}$ agit bien de sols non plastiques. Néanmoins, dans la maille C4, la couche se trouvant à $2 \mathrm{~m}$ de profondeur présente un $I_{c}$ supérieur à 2,6. Selon la procédure de Robertson, concernant cette couche, un calcul de I est refait pour n égal à 0,7 .

On rappelle ici, que l'analyse de risque de liquéfaction est principalement basée sur l'évaluation de la résistance sismique de la fondation (CRR) puis sa comparaison au rapport des contraintes cycliques (CSR) généré par un séisme. Dans le cas du barrage Sidi El Barrak, ce rapport, déduit à partir de l'équation 1, est calculé en considérant les valeurs de l'accélération maximale retenue suite à l'étude sismique. Cette accélération sera prise égale à $0,15 \mathrm{~g}$. La figure 17 montre la variation de CRR et CSR obtenue avant vibrocompactage en fonction de la profondeur de chaque maille. En se référant à ces graphiques, on remarque que pour la majorité des couches étudiées, le rapport CSR est nettement supérieur à la résistance du sol. Donc, la liquéfaction de la fondation du barrage est fortement probable dans le cas d'une accélération maximale de l'ordre de $0,15 \mathrm{~g}$. Après vibrocompactage, les valeurs de la résistance du sol à la liquéfaction CRR sont plus élevées que celles du rapport des contraintes cycliques CSR (Fig. 18). Ceci confirme, d'une part, l'amélioration de la résistance des terrains de la fondation et, d'autre part, l'efficacité de la technique de densification du sol. Une autre méthode d'identification des points à haut risque peut être utilisée. Elle consiste à préci ser les limites entre des échantillons qui peuvent subir une liquéfaction et les autres. Pour cela, il faut tracer le diagramme de CSR en fonction de $\mathrm{q}_{\mathrm{c} 1 \mathrm{~N}}$ (Fig. 19). Les points des essais après traitement sont placés à gauche de la courbe limite où le domaine est défini comme potentiellement liquéfiable. Les courbes obtenues après vibrocompactage permettent de constater que tous les points appartiennent à la zone non liquéfiable, ce qui traduit bien l'augmentation de la résistance du sol traité

De plus, les résultats d'une analyse traditionnelle du potentiel de liquéfaction s'appuyant sur le facteur
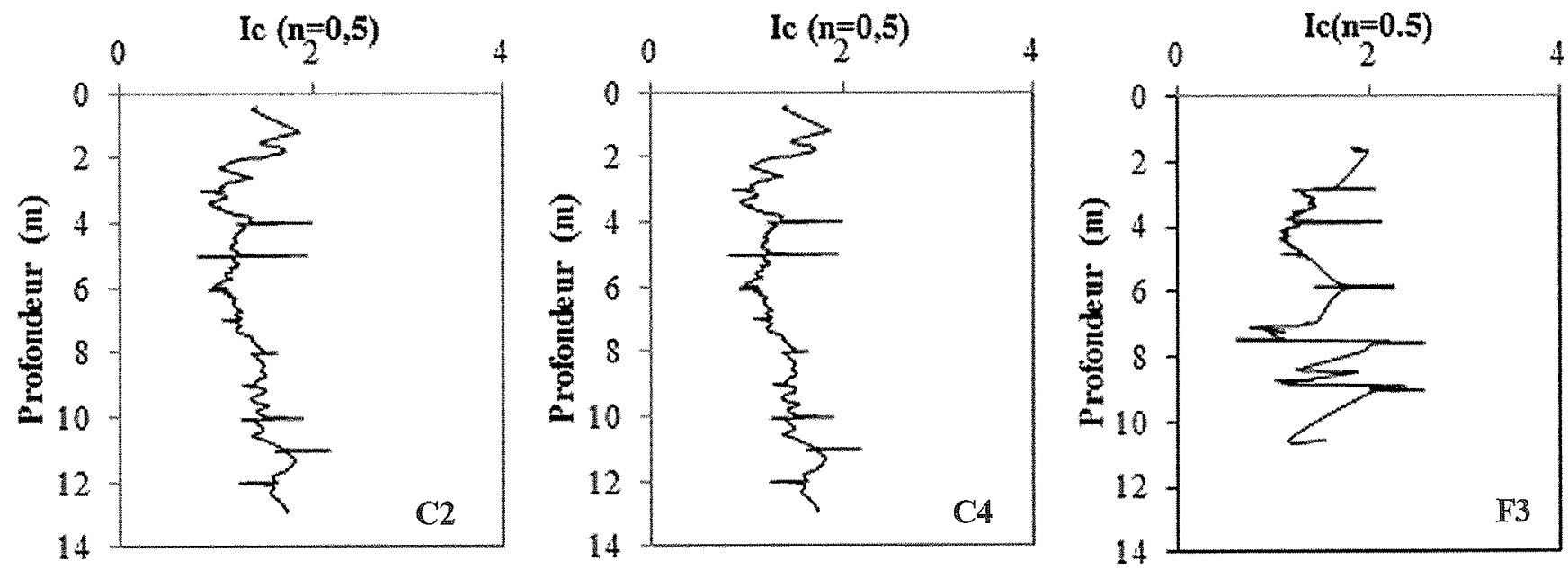

月6. 16 Profil de $\mathrm{I}_{c}$ de $\mathrm{C} 2, \mathrm{C} 4, \mathrm{~F} 3$.

Profile of $\mathrm{I}$ of $\mathrm{C} 2, \mathrm{C} 4, \mathrm{~F} 3$.
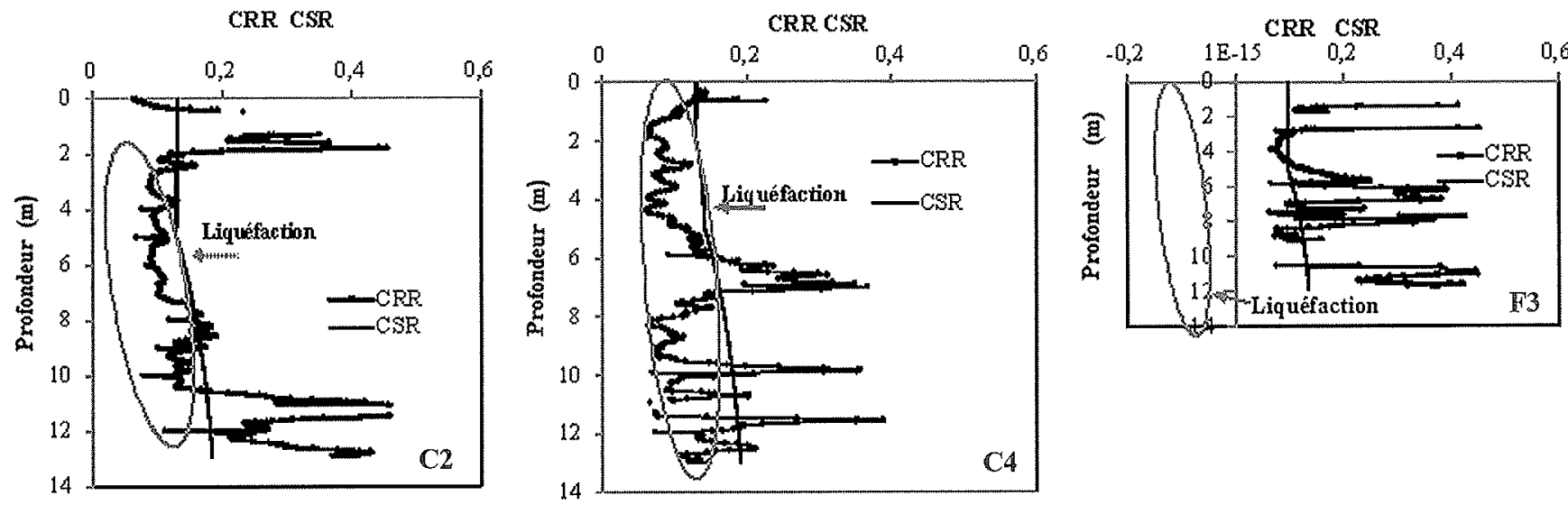

F6 17 CRR et CSR dans la maille C2, C4, F3 (avant vibrocompactage), CRR and CSR in mesh C2, C4, F3 (before vibrocompaction). 

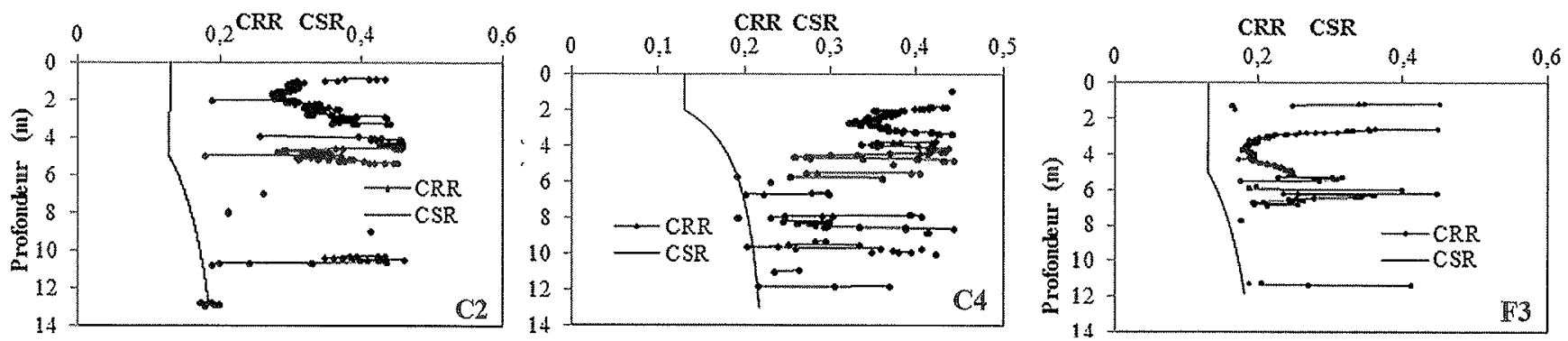

16. 18 Variation de CSR et CRR de C2, C4, F3. (après vibrocompactage). CRR and CSR in mesh C2, C4, F3 (after vibrocompaction).
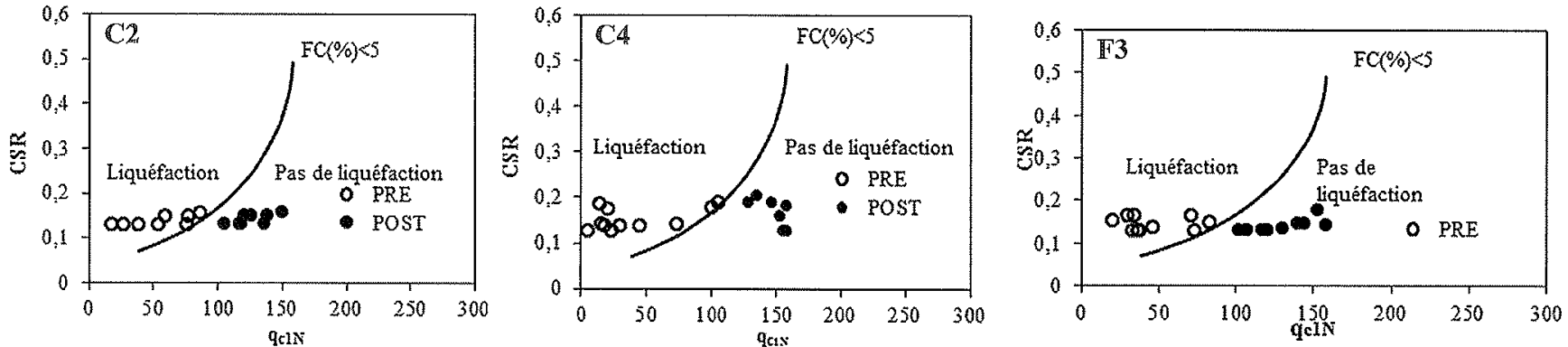

16. 19 CRR-q $\mathrm{q}_{\mathrm{c} 1 \mathrm{~N}}$ dans la maille C2, C4, F3.

$\mathrm{CRR}-\mathrm{q}_{\mathrm{C} 1 \mathrm{~N}}$ in the mesh $\mathrm{C} 2, \mathrm{C} 4, \mathrm{~F} 3$.

de sécurité FS, défini comme le rapport FS = CRR/CSR sont donnés sur la figure 20. D'après ces figures, les coefficients de sécurité indiquent un vrai potentiel de liquéfaction des couches où les valeurs calculées sont inférieures à 1 . Après vibrocompactage, les valeurs de FS sont supérieures à l'unité ce qui justifie l'absence de tout menace de liquéfaction.

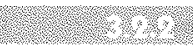

Méthode de Moss et al. (2006)

Le developpement des courbes enveloppes CRR a été effectué en considérant plusieurs autres paramètres tels que les propriétés des sols et les caracté- ristiques sismiques. Moss et al. (2006) ont cherché à représenter le CRR en se basant sur des méthodes statistiques bayésiennes. Ils ont proposé une famille de courbes limites calées pour differentes valeurs de probabilité (PL $=5,20,50,80$ et 95). Ainsi, l'évaluation de CRR à partir des mesures de CPT selon la méthode de Moss et al., (2006) est faite de la façon suivante :

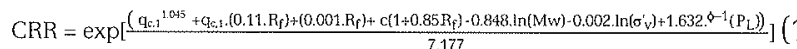

Avec $q_{c, 1}$ la résistance au cône modifiée, $M_{w}$ la magnitude du séisme de référence, $\Phi^{-1}$ la distribution normale cumulée inverse, $\mathrm{P}_{\mathrm{I}}$ la probabilité de liquéfaction dont l'expression est donnée par l'équation suivante :
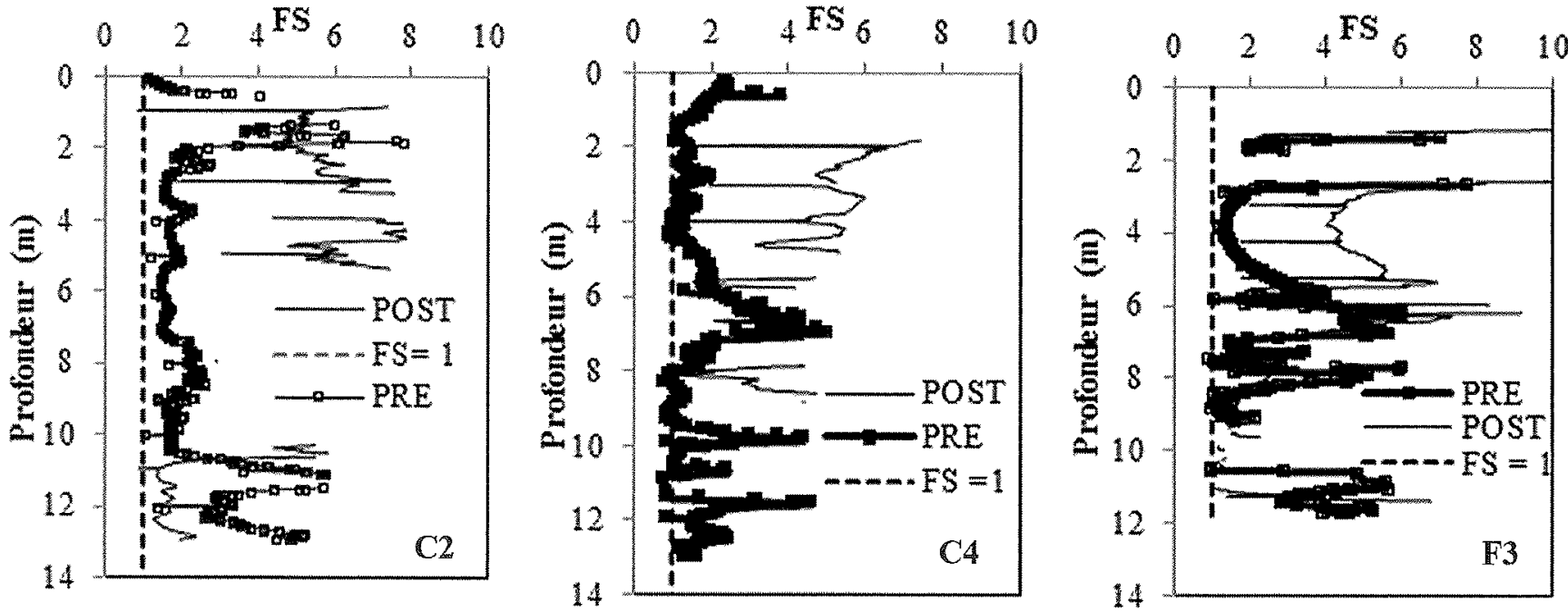

116. 20 Profil de FS de C2, C4, F3. Profile of FS of $\mathrm{C} 2, \mathrm{C} 4, \mathrm{~F} 3$. 
Dans le cas du présent barrage, les résultats des essais CPT effectués avant et après vibrocompactage ont été reproduits sur les graphiques reliant le rapport des contraintes cycliques (CSR) à la résistance à la pénétration modifiée $q_{c, 1}$ (Fig. 21). D’après ces courbes, on note que la fondation non traitée demeure en partie potentiellement liquéfiable puisque le nuage de points est placé à gauche des courbes enveloppes correspondantes aux différentes valeurs de probabilité. Par contre, après vibrocompactage, on note que tous les points sont décalés à droite des courbes limites dont la translation correspond à une augmentation des valeurs de la résistance corrigée.

De plus, cette approche sert à déterminer le facteur de sécurité FS de la fondation du barrage Sidi El Barrak (Figs. 22 et 23). Ces figures montrent qu'avant vibrocompactage les valeurs de FS sont inférieures à 1

ce qui témoigne l'existence de couches potentiellement liquéfiables. Par contre, après les travaux de densification du sol, il apparaît que le risque de liquéfaction diminue puisque $\mathrm{FS}>1$.

4

\section{Conclusion}

Dans le cas du barrage Sidi El Barrak, les résultats des essais in situ SPT et CPT et l'interprétation des différentes approches simplifiées utilisées sont conformes et similaires. La fondation du barrage est nettement envahie par des dépôts sableux perméables et le riscue sismique peut causer la liquéfaction des sables de l'ouvrage. Ainsi, un traitement par vibrocompactage a été choisi pour améliorer les caractéristiques mécaniques des sols de cette fondation. Après vibrocompactage, les résultats obtenus à partir des corrélations empiriques montrent que le risque est maîtrisé, et la fon-
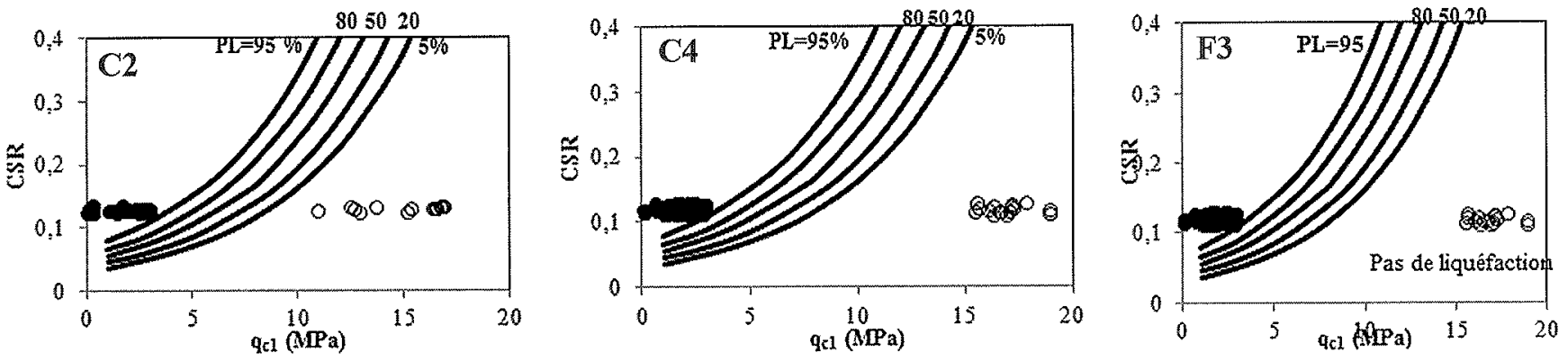

F16. 21. CSR en fonction de $q_{\mathrm{c} 1}$ dans $\mathrm{C} 2, \mathrm{C} 4, \mathrm{~F} 3$.

CSR as a function of $\mathrm{q}_{\mathrm{c} 1}$ in $\mathrm{C} 2, \mathrm{C} 4, \mathrm{~F} 3$
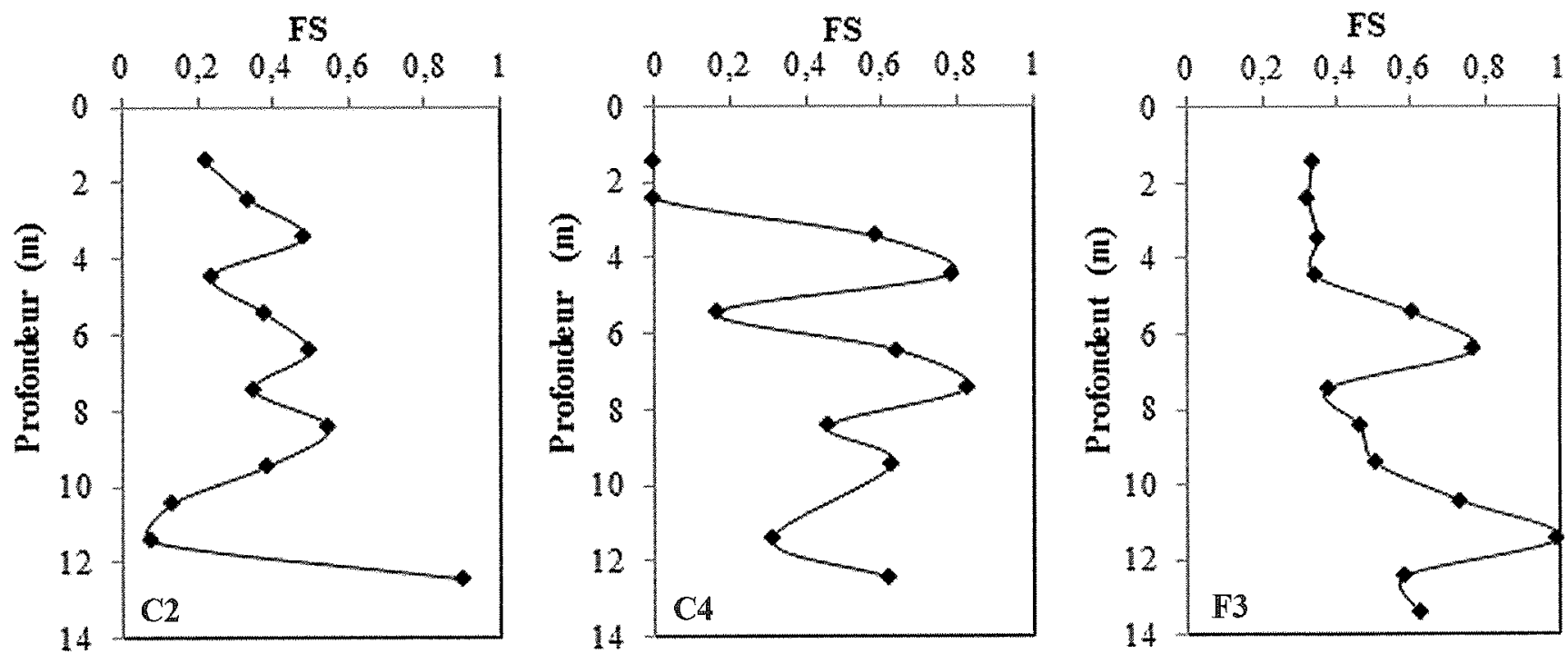

fG. 22 Variation de FS dans les mailles C2, C4 et F3 avant vibrocompactage. Variation of FS in meshes C2, C4 et F3 before vibrocompaction. 

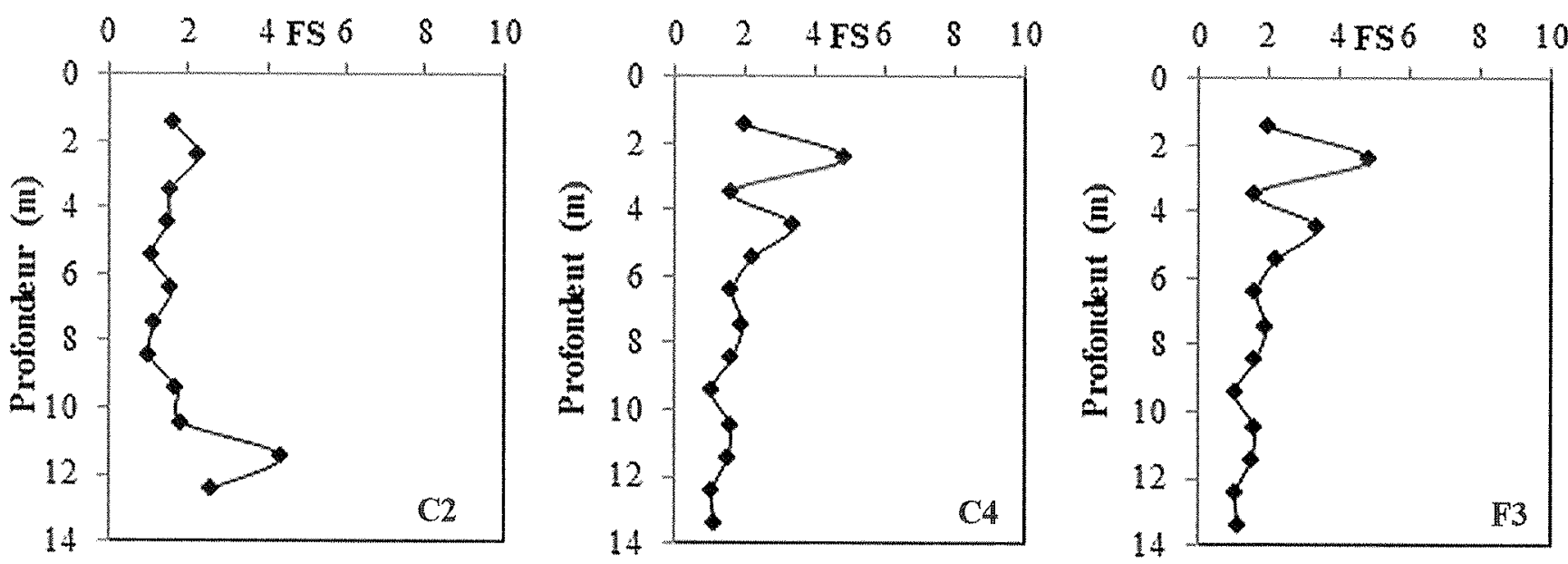

f16. 23 Variation de FS dans les mailles $\mathrm{C} 2$, C4 et F3 après vibrocompactage. Variation of FS in meshes C2, C4 et F3 after vibrocompaction

dation a été qualifiée. En effet, une amélioration des résistances des sols a été obtenue pour des séismes d'accélération maximale égale à $0,15 \mathrm{~g}$ où plusieurs points ont dépassé les conditions critiques de la liquéfaction. Les facteurs de sécurité calculés sont largement supérieurs à 1 au niveau des différentes mailles étudiées, et l'effet du vibrocompactage place la fonda- tion du barrage Sidi El Barrak en sécurité. Ainsi, une analyse systématique croisée des essais in situ SPT et CPT permet de poser un diagnostic pour un risque de liquéfaction des sols support. Cette étude doit donc servir de base à l'analyse d'autres sites rassemblant les mêmes conditions de risques.

\section{Bibliographie}

Blanchin M., Michalsky E.R., Dequidt O., Giafferi J.-L. - . La fondation du barrage de Sidi El Barrak (Tunisie). Journées nationales de Géotechnique et de Géologie de l'ingénieur, Nancy, oct. 2002.

Boulanger R.W., Idriss I.M. - Soil liquefaction during earthquake. Engineering monograph, EERI, California, USA, 2008, p. 266.

Boulanger R.W., Idriss I.M. - SPT-based liquefaction triggering procedure, 2010, p. 259.

Cetin K.O., Seed B., Kiureghian A.D., Tokimatsu K., Harder L.F., Kayen R.E., Moss R.E. - Standard Penetration TestBased Probabilistic and Deterministic Assessment of Seismic Soil Liquefaction Potential. Journal of Geotechnical and Geoenvironmental engineering, vol 130, $n^{\circ} 12,2004$, p. 1314-1340.

Corté J.-P. - Évaluation du risque de liquéfaction à partir des essais en place. In : Génie parasismique, Nantes, France, 1978 , p. 323-335.

El Ouni M.R., Bouassida M., Braja M.D. Vibrocompaction improvement of Tunisian liquefiable sands. Proceedings of the $17^{\text {th }}$ international conference on soil Mechanics and Geotechnical Engineering, 2009, 1-4.
Moss R.E.S., Seed R.B., Kayen R. E., Stewart J.P., Der Kiureghian A., Cetin K.O. - CPT-Based Probabilistic and Deterministic Assessment of In Situ Seismic Soil Liquefaction Potential. Journal of geotechnical and geoenvironmental engineering, 2006, p. 20.

Robertson P.K., Wride C. - Evaluating cyclic liquefaction potential using the cone penetration test. Canadian Geotechnical Journal, 1998, p. 442-459.

Robertson P.K., Wride C., Biggar K.W., Campanella R.G., Hofmann B.A., Hughes J.M.O., Küpper A., Woeller D.J. - Interpretation of in situ test results from the CANLEX sites. Canadian Geotechnical Journal, n 37, 2000, p. 505-529.

Seed B., Idriss I.M. - Simplified procedures for evaluating soil liquefaction potential. Proc. JSME, ASCE, vol. 97, SM9,1971, p. 1249-1273.

Seed B., Idriss I.M., Arango I. - Evaluation of liquefaction potential using field performance data. Journal Geotech, ASCE, vol. 109, n³ 3, 1983, p. 458-482.

Seed H.B., Tokimatsu K., Harder L.F. Chung R.M. - The influence of SPT Procedures in soil liquefaction. Journal of Geotechnical Engineering, ASCE, vol. $111, \mathrm{n}^{\circ} 12,1985$, p. $1425-1445$
Technical document - Drainage and treatment of the foundation of Sidi El Barrak dam (in French). Ministry of agriculture and Hydraulic resources of Tunisia, 1990.

Youd T.L., Andrus M., Idriss M. - Proceedings of the NCEER workshop on evaluation of liquefaction resistance of soils, 31 December, New York, USA, 1997, p. 1-40.

Youd T.L., Idriss I.M., Andrus R.D., Marcusson F., Robertson P.K., Seed R.B., Stokoe K.H. - Liquefaction resistance of soils : Summary report from the 1996 NCEER and 1998 NCEER/NSF workshops on evaluation of liquefaction resistance of soils. Journal of Geotechnical and Geoenviron. mental engineering, 2001 p. 817-833. 